\title{
Competition between sedimentation and phase coexistence of colloidal dispersions under gravity
}

\author{
Matthias Schmidt ${ }^{1,3}$, Marjolein Dijkstra ${ }^{1}$ and Jean-Pierre Hansen ${ }^{2,4}$ \\ ${ }^{1}$ Soft Condensed Matter, Debye Institute, Utrecht University, Princetonplein 5, 3584 CC Utrecht, \\ The Netherlands \\ 2 Dipartimento di Fisica, Universita degli Studi di Roma 'La Sapienza', Piazzale Aldo Moro, 2, \\ 00185 Roma, Italy
}

Received 17 April 2004, in final form 8 June 2004

Published 10 September 2004

Online at stacks.iop.org/JPhysCM/16/S4185

doi:10.1088/0953-8984/16/38/031

\begin{abstract}
After a brief review of the theory of sedimentation equilibria of colloidal systems, we consider the specific case of binary systems of hard sphere colloids and non-interacting polymer coils, the latter of vanishing buoyancy mass. The density profiles of the two components are calculated within density functional theory and using Monte Carlo simulations. Under appropriate conditions the profiles exhibit discontinuities or steeply varying regions associated with the interface separating colloid-rich and colloid-poor phases. The position of the interface is shown to be very sensitive to the strength of the gravitational field and, more surprisingly, to the total height $L$ of the suspension. Phase coexistence in the absence of gravity is shown to be entirely suppressed beyond a critical ratio of the height $L$ over the gravitational length of the colloids.
\end{abstract}

\section{Introduction}

Sedimentation profiles of macromolecules or colloidal dispersions subjected to gravity or centrifugal forces have been measured since the pioneering experiments of Jean Perrin in 1910 [1]. They contain a wealth of information about the osmotic equation of state [2] and phase behaviour [3] of interacting mesoscopic particles. Classical density functional theory (DFT) of inhomogeneous fluids [4] provides theoreticians with an ideal tool for the investigation of sedimentation equilibria. One of the open problems is understanding the influence of gravity, or more generally of external fields, on the phase diagram of colloidal systems, and in particular of binary systems, such as colloid-polymer mixtures, which will be the main focus of this paper. Phase equilibria of multi-component suspensions are examined under rather special

3 On leave from: Institut für Theoretische Physik II, Heinrich-Heine-Universität Düsseldorf, Universitätsstraße 1, D-40225 Düsseldorf, Germany.

4 On leave from: Department of Chemistry, University of Cambridge, Lensfield Road, Cambridge CB2 1EW, UK. 
conditions in sedimentation experiments, since the total volume and overall composition of the system are fixed [5], rather than the chemical potentials and pressure, as is the case for bulk, open systems connected to reservoirs of particles of each species. We shall see that these circumstances can affect the very existences of phase coexistence under gravity upon varying the total height of the sediment.

After a brief summary of the DFT of sedimentation equilibria in colloidal suspensions (section 2), we review some earlier theoretical work on sedimenting spheres, rods and plates in section 3, before investigating in more detail the case of colloid-polymer mixtures in section 4, with special emphasis on the influence of colloidal sedimentation on the bulk phase separation of these binary systems. Some concluding remarks will be made in section 5 .

\section{Sedimentation profiles and the osmotic equation of state}

Consider a dispersion of $v$ colloidal or macromolecular species in a suspending fluid, subjected to gravity. If $m_{\alpha}$ denotes the buoyant mass of species $\alpha(1 \leqslant \alpha \leqslant v)$, the effect of gravity may be characterized by the $v$ gravitational lengths $\xi_{\alpha}=k_{\mathrm{B}} T /\left(m_{\alpha} g\right)$, where $k_{\mathrm{B}} T$ is the thermal energy and $g$ the gravitational acceleration. The external field induces a vertical inhomogeneity in the suspension, which is characterized by the density profiles $\rho_{\alpha}(z)$, where $z$ is the vertical space coordinate. Under normal experimental conditions, the overall composition of the suspension is fixed, i.e.,

$$
\frac{1}{L} \int_{0}^{L} \rho_{\alpha}(z) \mathrm{d} z=n_{\alpha} ; \quad 1 \leqslant \alpha \leqslant v,
$$

where $L$ is the vertical height of the container and $n_{\alpha}$ is the overall number density of species $\alpha$. The profiles $\rho_{\alpha}(z)$ may be determined theoretically by minimizing the free energy functional (per unit area):

$F\left[\left\{\rho_{\alpha}(z)\right\}\right]=k_{\mathrm{B}} T \int_{0}^{L} \mathrm{~d} z \rho_{\alpha}(z)\left[\ln \left(\rho_{\alpha}(z) \Lambda_{\alpha}^{3}\right)-1\right]+F_{\mathrm{ex}}\left[\left\{\rho_{\alpha}\right\}\right]-\sum_{\alpha} \int_{0}^{L} \mathrm{~d} z \rho_{\alpha}(z) \Psi_{\alpha}(z)$.

The first term on the rhs of equation (2) is the familiar ideal contribution, $F_{\mathrm{id}}$, to the free energy $\left(\Lambda_{\alpha}\right.$ is an irrelevant length scale associated with species $\alpha$ ), the second term is the non-trivial excess contribution arising from the interactions between the suspended particles, while the last term accounts for the constraint (1) and the coupling to the external field:

$$
\Psi_{\alpha}(z)=\mu_{\alpha}-\phi_{\alpha}(z)=\mu_{\alpha}-m_{\alpha} g z
$$

where $\mu_{\alpha}$ is the Lagrange multiplier associated with constraint (1) (in an open system $\mu_{\alpha}$ would be the chemical potential of species $\alpha$ ) and $\phi_{\alpha}$ is the external potential acting on species $\alpha$. The density profiles are formally determined by the $v$ coupled Euler-Lagrange equations associated with the extremum conditions

$$
\frac{\delta F\left[\left\{\rho_{\alpha}\right\}\right]}{\delta \rho_{\beta}(z)}=k_{\mathrm{B}} T \ln \left(\rho_{\beta} \Lambda_{\beta}^{3}\right)+\frac{\delta F_{\mathrm{ex}}\left[\left\{\rho_{\alpha}\right\}\right]}{\delta \rho_{\beta}(z)}-\left(\mu_{\beta}-m_{\beta} g z\right)=0 .
$$

Total neglect of particle interactions (i.e. $F_{\mathrm{ex}}=0$ ) leads back to the familiar barometric formula $\rho_{\alpha}(z) \propto \exp \left(-z / \xi_{\alpha}\right)$. If the gravitational lengths $\xi_{\alpha}$ are much larger than the particle sizes $\sigma_{\alpha}$, the density profiles are expected to vary slowly over distances of the order of $\sigma_{\alpha}$, so the local density approximation (LDA) is applicable, whereby

$$
F_{\mathrm{ex}}\left[\left\{\rho_{\alpha}\right\}\right]=\int_{0}^{L} f_{\mathrm{ex}}\left(\left\{\rho_{\alpha}(z)\right\}\right) \mathrm{d} z
$$


where $f_{\mathrm{ex}}\left(\left\{\rho_{\alpha}\right\}\right)$ is the excess free energy density of the homogeneous multi-component dispersion as a function of the local densities $\rho_{\alpha}(z)$. The Euler-Lagrange equations (4) now reduce to

$$
\mu_{\beta}\left(\left\{\rho_{\alpha}(z)\right\}\right)=k_{\mathrm{B}} T \ln \left(\rho_{\beta}(z) \Lambda_{\beta}^{3}\right)+\frac{\partial f_{\mathrm{ex}}\left(\left\{\rho_{\alpha}(z)\right\}\right)}{\partial \rho_{\beta}(z)}=\mu_{\beta}-m_{\beta} g z .
$$

The 'local' chemical potential $\mu_{\beta}\left(\left\{\rho_{\alpha}(z)\right\}\right)$ is the sum of ideal and excess contributions, and equation (6) expresses local chemical equilibrium in an external field. Taking the derivative of equations (6) with respect to $z$, one arrives at the following set of coupled first-order differential equations for the density profiles [6]:

$$
\underline{\underline{M}}\left(\begin{array}{c}
\frac{\mathrm{d} \rho_{1}(z)}{\mathrm{d} z} \\
\vdots \\
\frac{\mathrm{d} \rho_{v}(z)}{\mathrm{d} z}
\end{array}\right)=-\left(\begin{array}{c}
1 / \xi_{1} \\
\vdots \\
1 / \xi_{v}
\end{array}\right),
$$

where the matrix elements of the $v \times v$ matrix $\underline{\underline{M}}$ are $M_{\alpha \beta}=\partial\left(\mu_{\alpha} /\left(k_{\mathrm{B}} T\right)\right) / \partial \rho_{\beta}$, calculated for $\rho_{\beta}=\rho_{\beta}(z)$. In the one-component case $(v=1)$, equation (7) reduces to

$$
\frac{\mathrm{d} \rho(z)}{\mathrm{d} z}=-\chi_{T}(\rho(z)) \frac{\rho(z)}{\xi}
$$

where $\chi_{T}(\rho)$ denotes the dimensionless osmotic compressibility $k_{\mathrm{B}} T(\partial \Pi / \partial \rho)^{-1}$ and $\Pi$ is the osmotic pressure of the suspension. The LDA equation (8) is equivalent to the macroscopic hydrostatic equilibrium condition:

$$
\frac{\mathrm{d} \Pi(\rho(z))}{\mathrm{d} z}=-m g \rho(z)
$$

Note that since $\chi_{T} \geqslant 0$, equation (8) can only yield monotonically decreasing density profiles $\rho(z)$, but this is no longer true of the multi-component generalization (7), which allows nonmonotonic behaviour of the coupled profiles $\rho_{\alpha}(z)$, as will be illustrated abundantly in the following sections. Note also that the LDA cannot account for particle layering at the bottom of the container. In fact, it yields coarse grained profiles $\bar{\rho}(z)$, 'smeared' over distances of the order of the particle diameter $\sigma_{\alpha}$ [2]. Finally, LDA entails a remarkable scale invariance, i.e. the profiles $\rho_{\alpha}\left(z / \xi_{\alpha}\right)$ as functions of the reduced altitude $z / \xi_{\alpha}$ depend only on the ratios $L / \xi_{\alpha}$, for any given overall composition $\left\{n_{\alpha}\right\}$. Integration of equation (9) gives

$$
\Pi(z) /\left(k_{\mathrm{B}} T\right)=\frac{1}{\xi} \int_{0}^{L} \rho\left(z^{\prime}\right) \mathrm{d} z^{\prime},
$$

so a measurement of $\rho(z)$ and subsequent elimination of the parameter $z$ between $\rho(z)$ and $\Pi(z)$ yields the osmotic equation of state $\Pi(\rho)$ of the homogeneous suspension [7].

\section{Sedimenting spheres, rods and plates}

The density profiles of suspensions of particles of various shapes can cross phase boundaries between phases of different bulk densities. Gravity-induced ordering is strikingly illustrated by the classic experiment of Pusey and van Megen [7] who were able to observe the coexistence of fluid and crystal phases of hard-sphere-like colloidal suspensions. The packing fractions of the coexisting phases agree perfectly with the predictions of computer simulations of bulk hard sphere systems. Complete density profiles can be measured by light scattering techniques [3] or using computer simulations [2].

Because of the finite resolution of the scattering experiments, profiles are naturally coarse grained (i.e. any rapid variation on the scale of the particle diameter is averaged out), so 
the LDA-based inversion procedure described at the end of the previous section is directly applicable. Piazza et al [3] were thus able to measure the osmotic pressure of hard-sphere-like colloids over a wide range of packing fractions, and their data in the fluid phase agree well with the quasi-exact Carnahan-Starling equation of state [8].

Gravity-induced ordering has also been predicted and observed for highly anisometric particles, such as long rods or thin plates, which form isotropic as well as nematic and columnar phases [9]. Onsager's celebrated theory of hard rod fluids [10] and its extensions are readily generalized to include sedimentation and for calculating density profiles of long hard rods under gravity [11]. The resulting profiles exhibit a sharp discontinuity as the isotropic-to-nematic boundary is crossed with decreasing altitude.

The 'inverse problem' was solved in Monte Carlo simulations of thin, disc-like platelets [12]: density profiles under gravity were inverted according to the prescription of equation (10) to extract the osmotic equation of state $\Pi(\rho)$. The results from a single simulation of the platelets subjected to gravity are in remarkable agreement with the classic data of Frenkel and Eppenga [13] from a series of simulations of the homogeneous system at various densities.

The remainder of this paper will deal with the sedimentation equilibrium of binary systems. The case of highly asymmetric binary mixtures of hard spheres was investigated in [6] within the LDA, i.e. by solving the coupled equations (7) (with $v=2$ ), and using the accurate equation of state of homogeneous hard sphere mixtures due to Mansoori et al [14]. Although binary hard sphere mixtures do not undergo a thermodynamically stable fluid-fluid bulk phase separation (the latter is pre-empted by freezing [15]), the calculated sedimentation profiles exhibit a strong size segregation, signalled by highly localized density profiles of the larger spheres. A diffuse slab of predominantly large spheres appears to float at a given altitude within an inhomogeneous fluid of mainly small spheres. The exact vertical location of the slab may be tuned by varying the mass ratio [6]. This observation provided the motivation for the study of the related system of colloid-polymer mixtures which will be reported on in the next section.

\section{Colloid sedimentation and phase coexistence of colloid-polymer mixtures}

We consider now binary suspensions of hard sphere colloids and non-adsorbing polymer coils, which have been abundantly investigated in recent years, both experimentally and theoretically (see [16] for a review and [17, 18] for recent experimental work). Under $\theta$-solvent conditions, the interactions between polymers may be neglected, and a popular model for describing mixtures of colloids and non-interacting polymers is that of Asakura and Oosawa [19] and Vrij [20] (AOV). This is a model of non-additive hard spheres with pair potentials:

$$
v_{\alpha \beta}(r)= \begin{cases}\infty, & r<\sigma_{\alpha \beta}, \\ 0, & r>\sigma_{\alpha \beta},\end{cases}
$$

where the indices $\alpha, \beta=\mathrm{c}$ (colloids) or $\mathrm{p}$ (polymers), $\sigma_{\mathrm{cc}}=\sigma_{\mathrm{c}}$ (colloid diameter), $\sigma_{\mathrm{pp}}=0$, $\sigma_{\mathrm{cp}}=\left(\sigma_{\mathrm{c}}+\sigma_{\mathrm{p}}\right) / 2$ and $\sigma_{\mathrm{p}}$ is twice the radius of gyration $R_{\mathrm{g}}$ of the polymer coils. Contrary to the case for additive hard spheres, the non-additive character of the model leads to phase separation into colloid-rich and colloid-poor fluid phases, for size ratios $q=\sigma_{\mathrm{p}} / \sigma_{\mathrm{c}} \gtrsim 0.4$. Complete phase diagrams have been established from theory [21,22] and computer simulations [23, 24]. The phase separation survives when polymer interactions are included, although the full phase diagram and in particular the location of the critical point in the $\left(\eta_{\mathrm{c}}, \eta_{\mathrm{p}}\right)$ plane are significantly modified [25]. Under most physical conditions, the ratio of buoyant masses $m_{\mathrm{p}} / m_{\mathrm{c}} \ll 1$ for comparable polymer and colloid sizes $\left(\sigma_{\mathrm{p}} \sim \sigma_{\mathrm{c}}\right)$, because polymer coils are fractal objects. It is hence reasonable to consider the case of massless polymer such that the polymer gravitational 
length $\xi_{\mathrm{p}} \rightarrow \infty$, while $\xi_{\mathrm{c}}$ remains finite. In other words, in the absence of colloids the polymer solution would remain homogeneous under gravity. The corresponding external potentials are given by

$$
\begin{aligned}
& v_{\mathrm{c}}^{\text {ext }}(z)= \begin{cases}m_{\mathrm{c}} g z, & \sigma_{\mathrm{c}} / 2<z<L-\left(\sigma_{\mathrm{c}} / 2\right), \\
\infty, & \text { otherwise },\end{cases} \\
& v_{\mathrm{p}}^{\text {ext }}(z)= \begin{cases}0, & \sigma_{\mathrm{p}} / 2<z<L-\left(\sigma_{\mathrm{p}} / 2\right), \\
\infty, & \text { otherwise. }\end{cases}
\end{aligned}
$$

The overall composition of the suspension is fixed, i.e. conditions (1) (with $\alpha=\mathrm{c}$ or $\mathrm{p}$ ) are satisfied. For a given composition, the key control parameter of the problem is the ratio $L / \xi_{\mathrm{c}}$. In the limit where $L$ and $\xi_{\mathrm{c}} \gg \sigma_{\mathrm{c}}$ the LDA is applicable. The excess free energy of the bulk colloid-polymer mixture in equation (5) is conveniently taken from the accurate free volume theory [22], which yields an analytic expression for $f_{\text {exc }}$. Within the LDA the profiles $\rho_{\mathrm{c}}(z)$ and $\rho_{\mathrm{p}}(z)$ depend only on the control parameter $L / \xi_{\mathrm{c}}$ when plotted versus the rescaled altitude $z / \xi_{\mathrm{c}}$ or $z / L$. Results of LDA calculations for a size ratio $q=1$ and overall packing fractions $\eta_{\mathrm{c}}=\pi n_{\mathrm{c}} \sigma_{\mathrm{c}}^{3} / 6=0.12$ and $\eta_{\mathrm{p}}=\pi n_{\mathrm{p}} \sigma_{\mathrm{p}}^{3} / 6=0.4$ are shown in figure 1 for several values of $L / \xi_{\mathrm{c}}$. In the absence of gravity $L / \xi_{\mathrm{c}}=0$ and the profiles reduce to step functions separating the colloid-rich 'liquid' phase $\left(\eta_{\mathrm{c}} \approx 0.23\right)$ from the colloid-poor 'gas' phase $\left(\eta_{\mathrm{p}} \approx 0.02\right)$. Increasing the ratio $L / \xi_{\mathrm{c}}$ can be achieved by considering either higher recipients $(L \uparrow)$ for a fixed gravitational length, or by reducing $\xi_{\mathrm{c}}$ (e.g. by changing the density of the suspending fluid) for fixed $L$. The latter situation is illustrated in figure 1(b).

Decreasing $\xi_{\mathrm{c}}$ amounts to increasing gravity so it is not surprising that the 'liquid-gas' interface shifts to lower altitudes. Note that the macroscopic LDA implies a discontinuous jump in the profile. The amplitude of the jump, i.e. the difference $\eta_{\mathrm{c}}^{l}-\eta_{\mathrm{c}}^{g}$ between the colloid packing fractions in the two coexisting phases, decreases with increasing $L / \xi_{\mathrm{c}}$ and shrinks to zero at about $L / \xi_{\mathrm{c}}=20.47$. In other words, for sufficiently strong gravity, phase coexistence is suppressed even well below critical conditions under field-free conditions. The same scenario for fixed $\xi_{\mathrm{c}}$ and increasing container height $L$ is illustrated in figure 1(a): for given overall packing fractions of the two species, the interface moves to higher altitudes when the container height $L$ increases, and vanishes beyond a critical height $L$, an observation which may appear intuitively less obvious. The polymer density profiles are shown in the two insets of figure 1: their partitioning between the two phases is complementary to that of the colloids, as expected.

The paths followed by the colloid-polymer mixture in the $\eta_{\mathrm{c}}-\eta_{\mathrm{p}}$ and $\eta_{\mathrm{c}}-\eta_{\mathrm{p}}^{\mathrm{r}}$ planes (where $\eta_{\mathrm{p}}^{\mathrm{r}}$ is the polymer packing fraction of a pure polymer reservoir that is in chemical equilibrium with the system) with increasing altitude are plotted relative to the phase separation binodals in figure 2 , for increasing values of the ratio $L / \xi_{\mathrm{c}}$. At the largest value of the latter, the path is seen to go through the critical point, so no phase separation will be observed for larger values of this ratio.

We consider next the case where no complete separation of length scales occurs, so that the macroscopic LDA is no longer exact. For a fixed container height $L=100 \sigma_{\mathrm{c}}$, we have used a fundamental measure theory (FMT) free energy functional [26, 27], which accounts properly for non-local effects, and reduces to the free energy of the free volume theory [22] in the homogeneous (field-free) limit. We have also carried out Monte Carlo (MC) simulations of the AOV model under gravity. Density profiles for increasing gravity, $\sigma_{\mathrm{c}} / \xi_{\mathrm{c}}=0.001,0.04,0.008$ and 0.2 (and hence $L / \sigma_{\mathrm{c}}=0.1,4,8,20$ ) are plotted in figure 3: the FMT (MC) results are shown in figures $3(\mathrm{a})$, (b). The two sets of data are seen to be in quantitative agreement, and to reproduce the trends predicted by the LDA (see figure 1(b)). However, both FMT and MC results show clear indications of layering at the bottom of the recipient, a feature on the scale 

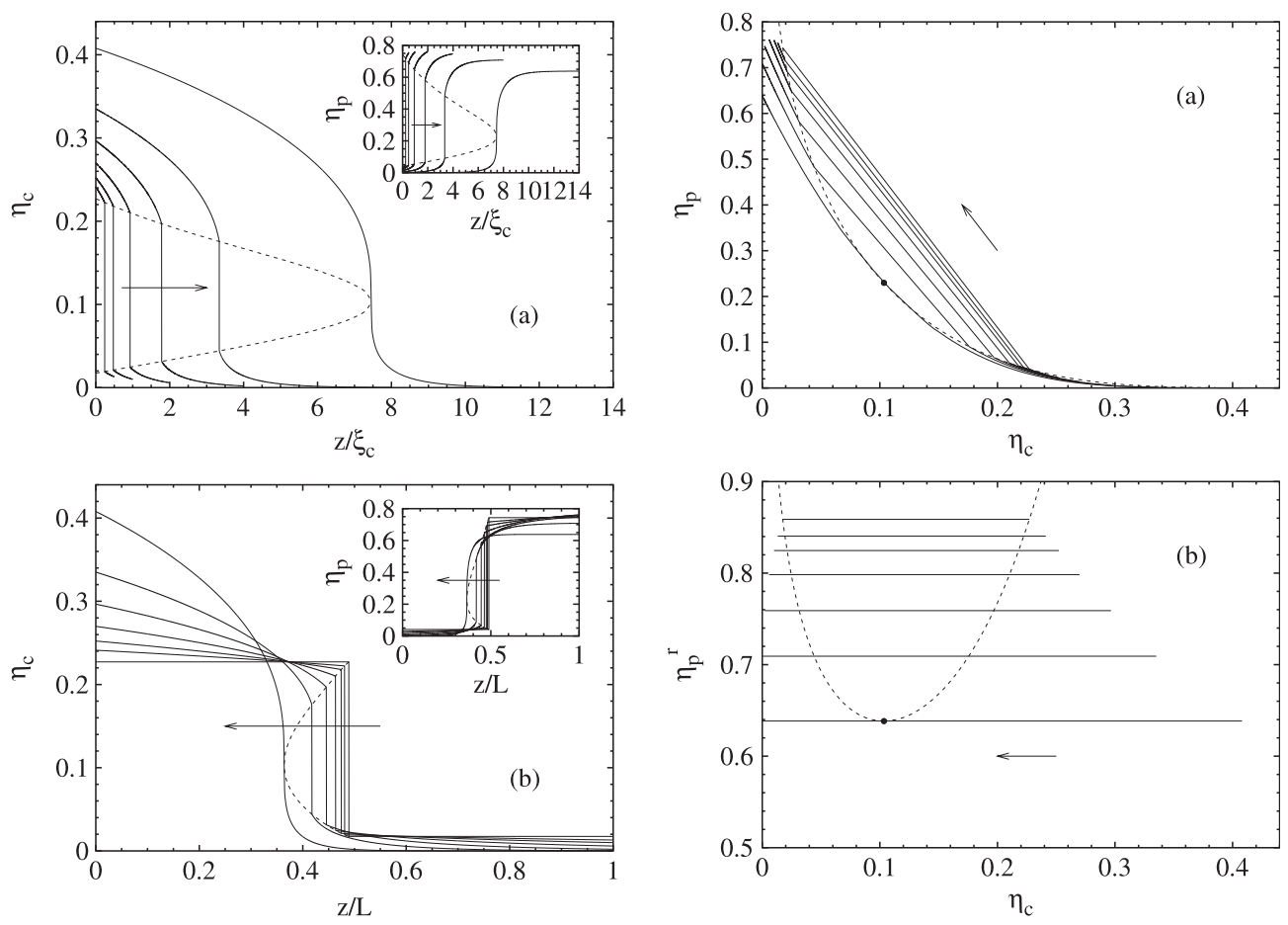

Figure 1. Dimensionless density profiles, $\eta_{\mathrm{c}}(z)=$ $\pi \rho_{\mathrm{c}}(z) \sigma_{\mathrm{c}}^{3} / 6$, as a function of the height variable scaled with the colloid gravitational length, $z / \xi_{\mathrm{c}}$, for size ratio $q=1$ and overall packing fractions $\eta_{\mathrm{c}}=0.12, \eta_{\mathrm{p}}=0.4$ (a) LDA results for different ratios of container height and gravitational length, $L / \xi_{\mathrm{c}}=0$ (on top of the vertical axes), 0.5, 1, 2, 4, 8, 20.47 (increasing as indicated by the arrow). The largest value corresponds to the case where the density jump (indicated by dashed curves) ends in a critical point. The inset shows the corresponding polymer profiles, $\eta_{\mathrm{p}}(z)=\pi \sigma_{\mathrm{p}}^{3} \rho_{\mathrm{p}}(z) / 6$. (b) Density profiles as a function of the height variable scaled with the height of

Figure 2. The bulk fluid-fluid demixing phase diagram of the AOV model as obtained from free volume theory for the size ratio $q=1$. Shown are the binodal (dashed curve) and sedimentation paths (full curves) for increasing altitude (in the direction of the arrow) corresponding to the density profiles in figure 1 . For each path the total packing fractions are $\eta_{\mathrm{c}}=0.12, \eta_{\mathrm{p}}=$ 0.4 , while $L / \xi_{\mathrm{c}}=0,0.5,1,2,4,8,20.47$ (from top to bottom). (a) The representation in the $\eta_{\mathrm{c}}-\eta_{\mathrm{p}}$ plane; (b) the representation in the $\eta_{\mathrm{c}}-\eta_{\mathrm{p}}^{\mathrm{r}}$ plane, where $\eta_{\mathrm{p}}^{\mathrm{r}}$ is the packing fraction in the polymer reservoir. the container, $z / L$.

of $\sigma_{\mathrm{c}}$ which cannot be accounted for by the LDA. Note also indications of wetting [28-32] of the upper wall of the recipient in the near homogeneous case $\sigma_{\mathrm{c}} / \xi_{\mathrm{c}}=0.001$. Finally, the density profiles are seen to vary rapidly at the interface, but there is no discontinuous jump as was the case for the LDA profiles in figure 1. This reflects the finite interfacial width on the scale of a few $\sigma_{\mathrm{c}}$. Since the calculations were carried out under conditions not far from the critical point of the bulk fluid-fluid demixing, the smoother variations of the MC profiles in the interfacial region compared to the FMT prediction reflect the differences in exact location of the critical point between the simulation results and the free volume theory [25, 30, 33, 34].

In order to highlight the qualitative differences between density profiles of fully miscible and phase-separating binary mixtures, we have carried out similar FMT [35] and MC calculations for a reference system, namely a binary system of additive hard spheres of identical diameters $(q=1)$, but with one of the species (playing the role of the polymer in the AOV model) being massless, i.e. not subject to gravitational effects. This system of course never phase separates, so the density profile is flat (except for layering oscillations near $z=0$ and 

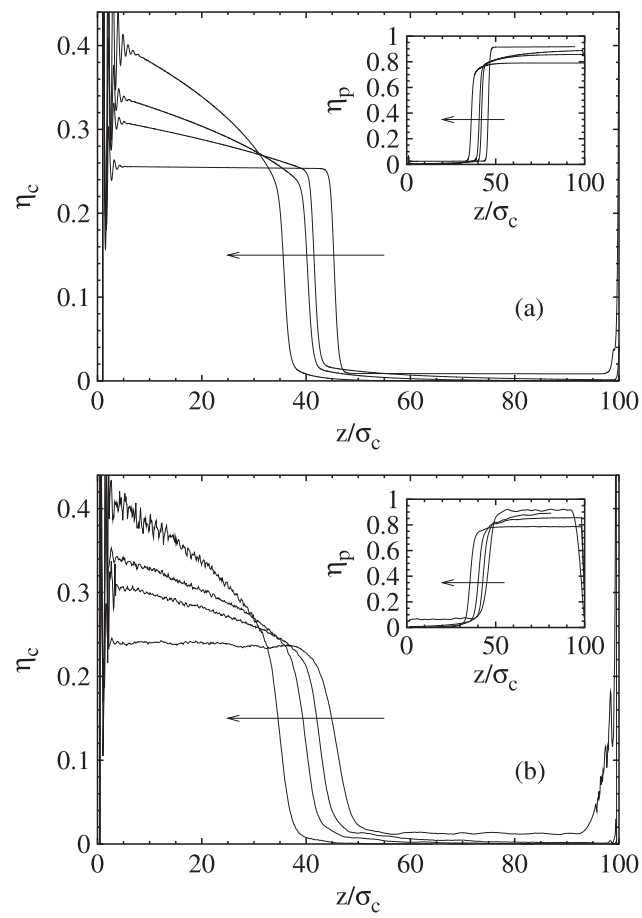

Figure 3. As figure 1, but for fixed container height $L=100 \sigma_{\mathrm{c}}$ and inverse gravitational lengths $\sigma_{\mathrm{c}} / \xi_{\mathrm{c}}=0.001,0.04,0.08,0.2$ (along the direction of the arrows). Shown are results from FMT (a) and MC (b) studies for $\eta_{\mathrm{c}}=0.12$ and $\eta_{\mathrm{p}}=0.5$.

$L$ ) in the absence of gravity. Figure 4 shows the FMT (4(a)) and MC (4(b)) results under conditions similar to those of figure 3. The density profiles of the two species are seen to steepen as gravity increases, but there is no trace of an interface between two phases, contrary to the AOV model results in figure 3.

Returning to the AOV model, we investigated the influence of the container height on the density profiles for a fixed value of the inverse gravitational length, namely $\sigma_{\mathrm{c}} / \xi_{\mathrm{c}}=0.08$. In figure 5 density profiles from FMT (5(a)) and MC (5(b)) are shown for increasing system size, $L / \sigma_{\mathrm{c}}=50,100$ and 200 , versus altitude in units of $\sigma_{\mathrm{c}}$ (or equivalently $\xi_{\mathrm{c}}$ ). The behaviour is seen to be similar to that predicted by the LDA for fixed $\xi_{\mathrm{c}}$ and increasing $L$ (see figure 1(a)). The same data are shown in figure 6 , with $z$ now rescaled by $L$. These profiles may be compared to the LDA results shown in figure 1(b), since the latter may be reinterpreted as profiles for a given $\xi_{\mathrm{c}}$ and increasing $L$. Clearly the position of the interface moves to lower scaled altitudes $z / L$ upon increasing the container height $L$.

The case where the polymer component is also subject to gravitational effects, i.e. $\xi_{p}$ is finite, gives rise to interesting re-entrant behaviour over a restricted range of ratios $\xi_{\mathrm{c}} / \xi_{\mathrm{p}}$, for which the sedimenting mixture crosses the bulk phase coexistence line twice. The resulting 'floating liquid' phase has been investigated elsewhere by DFT and MC techniques similar to those used in the present study for the case $\xi_{\mathrm{c}} / \xi_{\mathrm{p}}=0$ [36].

\section{Conclusions}

Sedimentation equilibria of colloidal systems allow phase coexistence lines to be crossed with varying altitude, i.e. an inhomogeneous suspension subjected to gravitational or centrifugal 

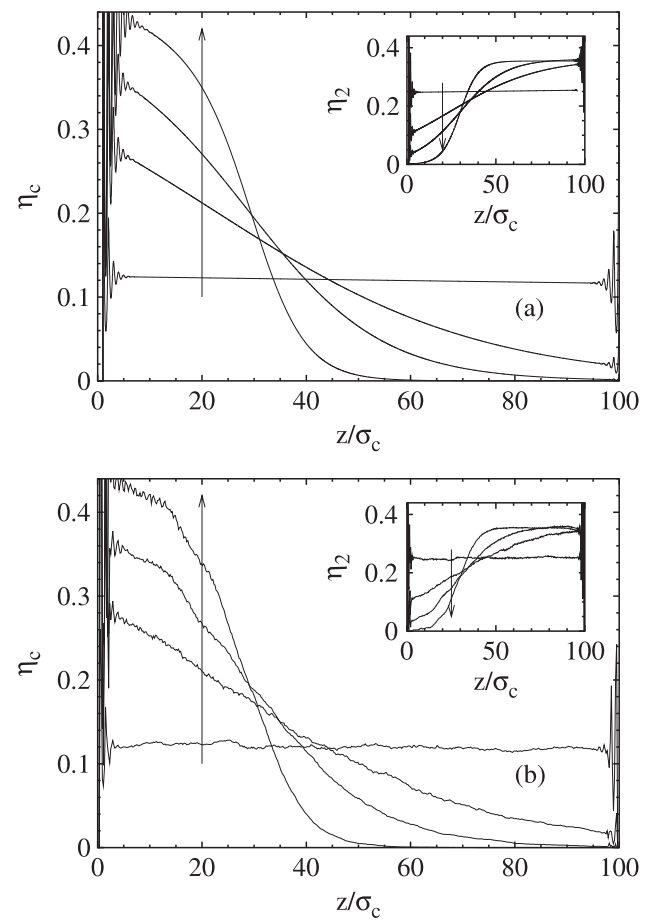

Figure 4. Density profiles of binary hard spheres obtained by formally replacing polymers of the AOV model with (additive) hard spheres of the same size (size ratio $q=1$ ). Only the colloid component sediment, the secondary hard sphere species (species 2), is massless. The packing fractions are $\eta_{\mathrm{c}}=0.12$ and $\eta_{2}=0.25$, and the scaled inverse gravitational height is $\sigma_{\mathrm{c}} / \xi_{\mathrm{c}}=0.001,0.04,0.08,0.2$ (as indicated by the arrows). Results are shown from FMT (a) and MC simulation (b).

fields can exhibit coexisting phases of different symmetry or composition even if the homogeneous system of identical overall composition exists in a single phase. Gravity-induced density gradients have been exploited, both experimentally and theoretically, to determine the osmotic equation of state and the phase diagram of colloidal systems for many years, and some representative examples of this work have been briefly reviewed in sections 2 and 3 . The main focus of this paper was on binary systems of hard sphere colloids and non-interacting, (buoyancy) massless polymers. We have investigated the influence of gravity on the coexistence between colloid-rich and colloid-poor phases. Except in the vicinity of the critical consolute point, phase coexistence in this system is characterized by large discontinuities in composition, and hence by steep variations of the density profiles in the interfacial region separating the two phases. The present LDA, FMT and MC calculations show that, for a given overall composition, the position of the interface is sensitive to the gravitational strength, and more specifically to the ratio of container height over gravitational length, $L / \xi_{\mathrm{c}}$. In particular we find the somewhat surprising result that the position of the interface moves to lower rescaled altitudes $z / L$ when the height $L$ of the container increases. This sensitivity of the interface between coexisting phases to gravity and container height implies that some care has to be taken when information on phase coexistence of homogeneous (field-free) systems is to be extracted from experimental observations of phase separation under gravity. In particular, for sufficiently large ratios $L / \xi_{\mathrm{c}}$, phase coexistence may be suppressed altogether in the sediment. 

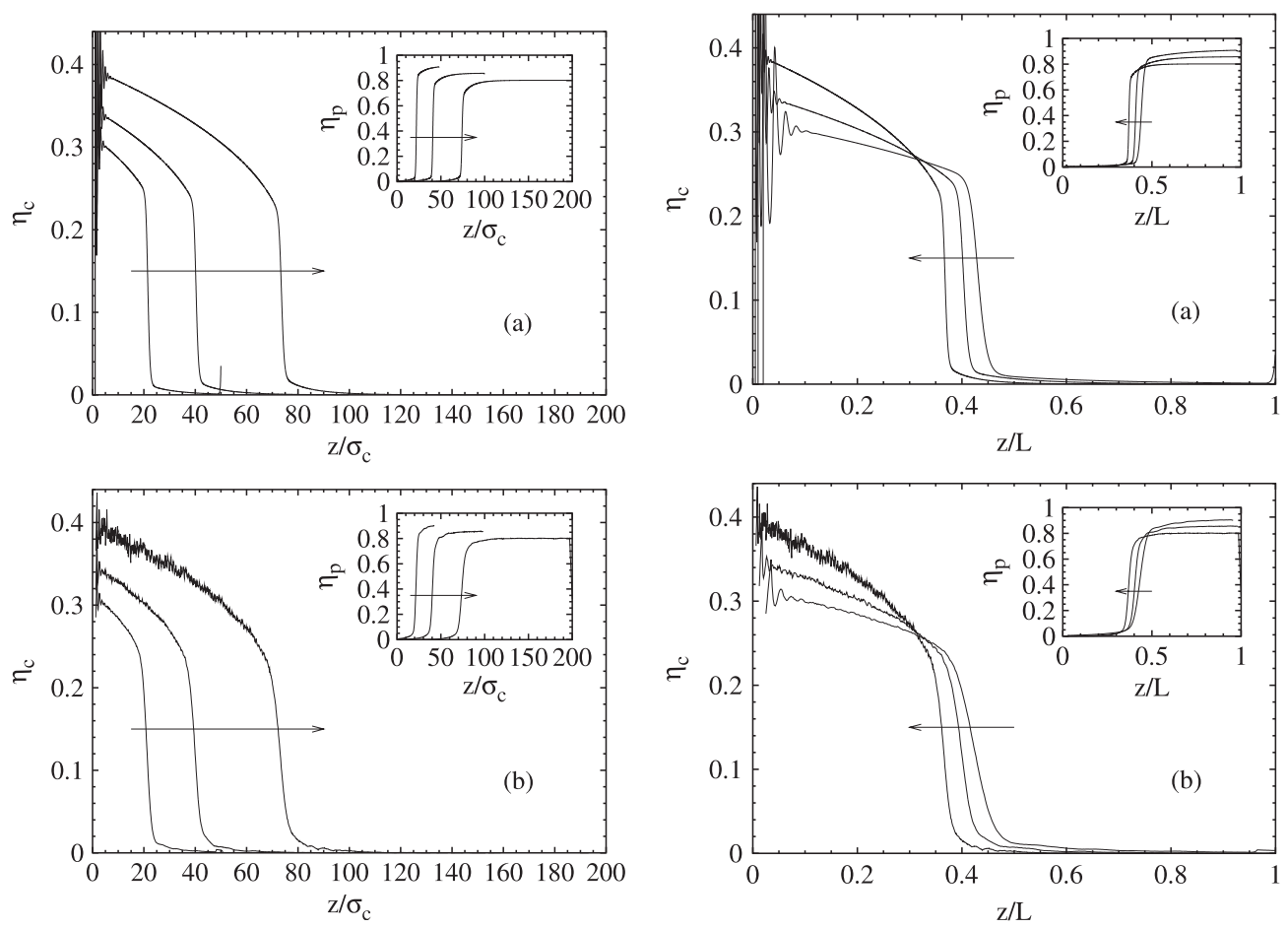

Figure 5. As figure 3, but for a fixed (inverse) gravitational length $\sigma_{\mathrm{c}} / \xi_{\mathrm{c}}=0.08$ and increasing system size $L / \sigma_{\mathrm{c}}=50,100,200$ (as indicated by the arrows). Shown are results from FMT (a) and MC simulations (b).

Figure 6. As figure 5, but as a function of the altitude scaled by the total container height, $z / L$. Clearly the position of the interface moves to smaller scaled altitudes upon increasing $L$.

All our considerations rely on equilibrium states; the timescale for reaching true equilibrium in an experimental colloid-polymer set-up may be considerable [37].

\section{Acknowledgments}

JPH is grateful to Giovanni Ciccotti, Giorgio Parisi and to INFM for their hospitality at La Sapienza University in Rome. MS thanks Dirk Aarts for many inspiring discussions. The work of MS is part of the research programme of the Stichting voor Fundamenteel Onderzoek der Materie (FOM), which is financially supported by the Nederlandse Organisatie voor Wetenschappelijk Onderzoek (NWO). We acknowledge support from the SFB-TR6 'Physics of colloidal dispersions in external fields' of the Deutsche Forschungsgemeinschaft (DFG).

\section{References}

[1] Perrin J 1910 J. Physique 95

[2] Biben T, Hansen J-P and Barrat J L 1993 J. Chem. Phys. 987330

[3] Piazza R, Bellini T and Degiorgio V 1993 Phys. Rev. Lett. 714267

[4] Evans R 1992 Fundamentals of Inhomogeneous Fluids ed D Henderson (New York: Dekker) chapter 3, p 85

[5] Bodnár J and Oosterbaan W D 1997 J. Chem. Phys. 1067777

[6] Biben T and Hansen J 1993 Mol. Phys. 80853

[7] Pusey P N and van Megen W 1984 Nature 320340 
[8] Carnahan N F and Starling K E 1969 J. Chem. Phys. 51635

[9] Wensink H H and Lekkerkerker H N W 2004 Europhys. Lett. 66125

[10] Onsager L 1949 Ann. New York Acad. Sci. 51627

[11] Allen R J, Goulding D and Hansen J-P 1999 Phys. Chem. Commun. 7

[12] Dijkstra M, Hansen J-P and Madden P A 1995 Phys. Rev. Lett. 752236

[13] Frenkel D and Eppenga R 1982 Phys. Rev. Lett. 491089

[14] Mansoori G A, Carnahan N F, Starling K E and Leyland T W 1975 J. Chem. Phys. 541523

[15] Dijkstra M, van Roij R and Evans R 1999 Phys. Rev. E 595744

[16] Poon W C K 2002 J. Phys.: Condens. Matter 14 R859

[17] Aarts D G A L, van der Wiel J H and Lekkerkerker H N W 2003 J. Phys.: Condens. Matter 15 S245

[18] Aarts D G A L and Lekkerkerker H N W 2004 Proc. CODEF Conf. (2004); J. Phys.: Condens. Matter 16 S4231

[19] Asakura S and Oosawa F 1954 J. Chem. Phys. 221255

[20] Vrij A 1976 Pure Appl. Chem. 48471

[21] Gast A P, Hall C K and Russell W B 1983 J. Colloid Interface Sci. 96251

[22] Lekkerkerker H N W, Poon W C K, Pusey P N, Stroobants A and Warren P B 1992 Europhys. Lett. 20559

[23] Meijer E J and Frenkel D 1994 J. Chem. Phys. 1006873

[24] Dijkstra M, Brader J M and Evans R 1999 J. Phys.: Condens. Matter 1110079

[25] Bolhuis P G, Louis A A and Hansen J P 2002 Phys. Rev. Lett. 89128302

[26] Schmidt M, Löwen H, Brader J M and Evans R 2000 Phys. Rev. Lett. 851934

[27] Schmidt M, Löwen H, Brader J M and Evans R 2002 J. Phys.: Condens. Matter 149353

[28] Brader J M, Evans R, Schmidt M and Löwen H 2002 J. Phys.: Condens. Matter 14 L1

[29] Brader J M, Evans R and Schmidt M 2003 Mol. Phys. 1013349

[30] Dijkstra M and van Roij R 2002 Phys. Rev. Lett. 89208303

[31] Aarts D G A L, Dullens R P A, Lekkerkerker H N W, Bonn D and van Roij R 2003 J. Chem. Phys. 1201973

[32] Wessels P P F, Schmidt M and Löwen H 2004 Proc. CODEF Conf. (2004); J. Phys.: Condens. Matter 16 S4169

[33] Vink R L C and Horbach J 2003 Preprint cond-mat/0310404

[34] Vink R L C and Horbach J 2004 Proc. CODEF Conf.; J. Phys.: Condens. Matter 16 S3807

[35] Rosenfeld Y 1989 Phys. Rev. Lett. 63980

[36] Schmidt M, Dijkstra M and Hansen J-P 2004 at press

[37] Aarts D G A L, private communication 